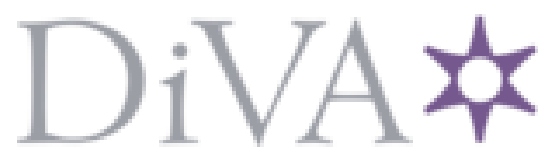

http://www.diva-portal.org

\title{
Postprint
}

This is the accepted version of a paper presented at IEEE International Symposium on Information Theory (ISIT).

Citation for the original published paper:

Girnyk, M., Vehkaperä, M., Rasmussen, L. (2014)

Asymptotic Properties of Dual-Hop AF Relay MIMO Communication Systems.

In: Proceedings of IEEE International Symposium on Information Theory (ISIT)

N.B. When citing this work, cite the original published paper.

Permanent link to this version:

http://urn.kb.se/resolve?urn=urn:nbn:se:kth:diva-149871 


\section{Asymptotic Properties of Dual-Hop AF Relay MIMO Communication Systems}

\author{
Maksym A. Girnyk \\ School of Electrical Engineering \\ and the ACCESS Linnaeus Centre, Department of Signal Processing and Acoustics, \\ KTH Royal Institute of Technology, \\ Stockholm, Sweden \\ Email: maksym.girnyk@ee.kth.se
}

\author{
Lars K. Rasmussen \\ School of Electrical Engineering \\ and the ACCESS Linnaeus Centre, \\ KTH Royal Institute of Technology, \\ Stockholm, Sweden \\ Email: lars.rasmussen@ieee.org
}

\begin{abstract}
The present paper studies the asymptotic performance of dual-hop amplify-and-forward multiple-input multipleoutput relay communication systems. In the corresponding setup, a relay amplifies the signal received from a source, retransmitting it towards a destination, while the direct source-destination link is absent. Ergodic achievable rates under separate decoding, along with the average bit error rate under various detection schemes are derived in the regime where the number of antennas at each terminal grows without bound. To overcome the mathematical difficulty of averaging over both channel realizations and input signals we apply large-system analysis based on the replica method from statistical physics. The validity of the large-system analysis is further verified through Monte Carlo simulations, providing particularly good accuracy at low SNR.
\end{abstract}

\section{INTRODUCTION}

Multiple-input multiple-output (MIMO) relaying has been proved a promising technology that allows for reliable communication at increased coverage and data rates [1]. Understanding the fundamental limits of the relay channel has therefore become one of the recent research challenges. The relay MIMO channel (depicted in Fig. 1) - consisting of a source $(S)$, a relay $(R)$ and a destination $(D)$ - is a fundamental building block for complex cooperative networks [2], [3]. It has therefore been regarded as an important milestone in the research efforts within the field.

The relays in a cooperative network may realize different cooperative strategies. For instance, a regenerative strategy (e.g., decode-and-forward [4]) involves decoding of the noisy signal from the source, re-encoding the underlying message and retransmitting it upstream. A non-regenerative strategy (e.g., amplify-and-forward (AF) [2]) involves simple amplification of the received noisy signal and subsequent retransmission towards the destination. Non-regenerative relaying, being simple in implementation and independent of the modulation schemes at the source terminal, is of particular interest.

In fading environment, the achievable data rate of such cooperative communication systems is measured by the endto-end ergodic mutual information (MI). Hence, performance evaluation requires averaging over the channel randomness. This, in general, prevents one from having closed-form expressions and motivates the need for alternative approaches to

The present research was supported by the Swedish Research Council.

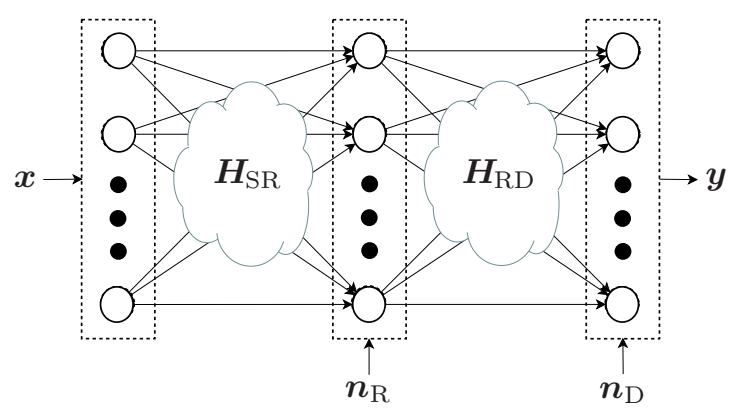

Fig. 1. Multiple-input multiple-output communication system.

characterize the achievable rates. However, large-system techniques have been introduced [5] to overcome such difficulties. In this regime, the capacity of a generic MIMO system has been derived in [6]. In the AF relay setting, end-to-end ergodic MI has been characterized in [7], [8] for arbitrary inputs.

For uncoded systems, reliability is evaluated in terms of uncoded average bit error rate (BER), which too yields no closed-form expression for relay MIMO scenario with fading. In [9], the instantaneous BER of a BPSK constellation is derived and the average performance is obtained through subsequent Monte Carlo simulation. Most of the existing work on closed-form average BER in AF MIMO setting is limited either to one-antenna terminals [10], or to bounds on the BER performance [11]. Furthermore, the large-system BER performance of the MMSE detector has been analyzed in [7]. However, the problem in its general formulation, accounting for other conventional detectors, remains open.

The present paper investigates the performance of a dualhop AF relay MIMO communication system. Our analysis is based on the RS ansatz of the replica method [12] from statistical physics and provides a framework for efficient performance analysis of AF MIMO relay systems under general conditions. The analysis leads to large-system approximations for the achievable ergodic rate and uncoded average BER, which become exact when the numbers of antennas at all terminals grow large without bound at constant ratios. Our results partially reduce to those presented in [6], [7], as well as [8] for two hops. 


\section{System Model}

Consider a two-hop AF MIMO relay channel depicted in Fig. 1. Communication is carried out in a half-duplex mode over two time-slots. The output of the relay channel reads as

$$
\boldsymbol{y}=\sqrt{\beta} \boldsymbol{H}_{\mathrm{RD}} \boldsymbol{H}_{\mathrm{SR}} \boldsymbol{x}+\sqrt{\beta} \boldsymbol{H}_{\mathrm{RD}} \boldsymbol{n}_{\mathrm{R}}+\boldsymbol{n}_{\mathrm{D}},
$$

where $\boldsymbol{x}$ is the signal vector transmitted from the source, such that $\mathrm{E}\left\{\boldsymbol{x}_{0} \boldsymbol{x}_{0}^{\mathrm{H}}\right\}=\mathbf{I}_{M} ; \boldsymbol{H}_{\mathrm{SR}} \in \mathbb{C}^{L \times M}$ is the source-relay channel matrix, $\boldsymbol{H}_{\mathrm{RD}} \in \mathbb{C}^{N \times L}$ is the relay-destination channel matrix, $\boldsymbol{n}_{\mathrm{R}} \sim \mathcal{C N}\left(\mathbf{0}_{L \times L}, \mathbf{I}_{L}\right)$ and $\boldsymbol{n}_{\mathrm{D}} \sim \mathcal{C N}\left(\mathbf{0}_{N \times N}, \mathbf{I}_{N}\right)$ are standard complex additive Gaussian noise vectors at the relay and destination, respectively. Furthermore, $\rho_{\mathrm{SR}}>0$ and $\rho_{\mathrm{RD}}>0$ are the transmit signal-to-noise ratios (SNRs) of the source-relay and relay-destination channels, respectively. Hereafter, we consider uncorrelated channel matrices, whose elements are i.i.d. and distributed as $\left[\boldsymbol{H}_{\mathrm{SR}}\right]_{l, m} \sim$ $\mathcal{C N}\left(0, \rho_{\mathrm{SR}} / M\right),\left[\boldsymbol{H}_{\mathrm{RD}}\right]_{n, l} \sim \mathcal{C N}\left(0, \rho_{\mathrm{RD}} / L\right), \forall m, l, n$. The power normalization factor $\beta$ is chosen to satisfy the longterm power constraint at the relay, i.e., $\beta \mathrm{E}\left\{\operatorname{tr}\left\{\boldsymbol{y}^{\mathrm{H}}\right\}\right\} \leq L$.

For compactness, denote $\boldsymbol{H} \triangleq \sqrt{\beta} \boldsymbol{H}_{\mathrm{RD}} \boldsymbol{H}_{\mathrm{SR}}$ and $\overline{\boldsymbol{H}} \triangleq$ $\sqrt{\beta} \boldsymbol{H}_{\mathrm{RD}}$, so that the effective channel reads

$$
\boldsymbol{y}=\boldsymbol{H} \boldsymbol{x}+\overline{\boldsymbol{H}} \boldsymbol{n}_{\mathrm{R}}+\boldsymbol{n}_{\mathrm{D}} .
$$

Meanwhile, we assume that the instantaneous channel state information (CSI), denoted as $\mathcal{H}=\{\boldsymbol{H}, \overline{\boldsymbol{H}}\}$, is known at the receiver. The conditional distribution of channel (2) is thus

$$
p(\boldsymbol{y} \mid \boldsymbol{x}, \mathcal{H})=\frac{1}{\pi^{N}} \mathrm{E}_{\boldsymbol{n}_{\mathrm{R}}}\left\{\mathrm{e}^{-\left\|\boldsymbol{y}-\boldsymbol{H} \boldsymbol{x}-\overline{\boldsymbol{H}} \boldsymbol{n}_{\mathrm{R}}\right\|^{2}}\right\},
$$

and the ergodic end-to-end MI is given by

$$
I(\boldsymbol{y} ; \boldsymbol{x})=\mathrm{E}_{\boldsymbol{y}, \mathcal{H}}\left\{\ln \left(\frac{p(\boldsymbol{y} \mid \boldsymbol{x}, \mathcal{H})}{\mathrm{E}_{\boldsymbol{x}}\{p(\boldsymbol{y} \mid \boldsymbol{x}, \mathcal{H})\}}\right)\right\} .
$$

Although (4) represents the maximum achievable rate of a relay MIMO system, it involves optimal joint detection and decoding (JDD), which in practice may be prohibitively complex. A more plausible alternative involves joint detection, followed by a bank of independent decoders and is referred here as separate decoding (SD). In this case, the detector estimates the symbol vector based on the generalized posterior mean estimator (GPME), given by

$$
\left\langle\boldsymbol{x}^{\prime}\right\rangle_{q}=\int \boldsymbol{x}^{\prime} \frac{q\left(\boldsymbol{x}^{\prime}\right) q\left(\boldsymbol{y} \mid \boldsymbol{x}^{\prime}, \mathcal{H}\right)}{\int q\left(\boldsymbol{x}^{\prime}\right) q\left(\boldsymbol{y} \mid \boldsymbol{x}^{\prime}, \mathcal{H}\right) \mathrm{d} \boldsymbol{x}^{\prime}} \mathrm{d} \boldsymbol{x}^{\prime},
$$

where subscript $q$ reflects the fact that the receiver uses some postulated channel law $q\left(\boldsymbol{y} \mid \boldsymbol{x}^{\prime}, \mathcal{H}\right)$ and distribution $q\left(\boldsymbol{x}^{\prime}\right)$ of postulated inputs $\boldsymbol{x}^{\prime}$. The GPME may be suboptimal if the latter do not match conditional density $p(\boldsymbol{y} \mid \boldsymbol{x}, \mathcal{H})$ and the prior distribution $p(\boldsymbol{x})$ of the actual channel. However, as it was shown in [5], any conventional detector can be regarded as the GPME, optimal for the postulated channel law $q\left(\boldsymbol{y} \mid \boldsymbol{x}^{\prime}, \mathcal{H}\right)$. It was further shown that in order to capture suboptimality of a variety of detectors of interest it suffices to postulate a channel with a mismatch only in the noise variance $\sigma^{2}$ and prior distribution $q\left(\boldsymbol{x}^{\prime}\right)$, i.e.,

$$
\boldsymbol{y}=\boldsymbol{H} \boldsymbol{x}^{\prime}+\overline{\boldsymbol{H}} \boldsymbol{n}_{\mathrm{R}}^{\prime}+\boldsymbol{n}_{\mathrm{D}}^{\prime},
$$

where $\boldsymbol{x}^{\prime}$ is the postulated channel input vector, and $\boldsymbol{n}_{\mathrm{R}}^{\prime} \sim$ $\mathcal{C N}\left(\mathbf{0}_{L \times L}, \sigma^{2} \mathbf{I}_{L}\right)$ and $\boldsymbol{n}_{\mathrm{D}}^{\prime} \sim \mathcal{C N}\left(\mathbf{0}_{N \times N}, \sigma^{2} \mathbf{I}_{N}\right)$ are postulated noise vectors with variance $\sigma^{2}$.

Apart from individually optimal detector, this paper considers also the three basic linear detectors, namely matched filter $(\mathrm{MF})$, zero forcing $(\mathrm{ZF})$ detector, and the linear minimum mean square error (MMSE) detector. In general, average performance of these detection schemes over fading channels has no closed-form expression. The analysis is further complicated by the fact that in the AF relay setting channel matrix $\boldsymbol{H}$ is a product of the channel matrices of the two hops, yielding a distribution of the entries, which is not i.i.d. In the following section, we present a framework leading to a large-system approximation for the performance of the AF relay MIMO system (in terms of both average BER and achievable rate under SD), which becomes tight as the system size grows large.

\section{As YMPtotic Properties}

To begin with, we define the large-system limit (LSL) as the regime where the number of antennas at all terminals grows large without a bound at constant rates, that is, $M, L, N \rightarrow$ $\infty, \alpha_{\mathrm{SR}}=L / M=$ const, $\alpha_{\mathrm{RD}}=N / L=$ const, $\alpha_{\mathrm{SD}}=$ $N / M=$ const. The main result is then summarized below.

Claim 1. For the channel model in (2) let $x_{m}, x_{m}^{\prime}$, and $\left\langle x_{m}^{\prime}\right\rangle_{q}$ denote the mth entries of $\boldsymbol{x}, \boldsymbol{x}^{\prime}$ and $\left\langle\boldsymbol{x}^{\prime}\right\rangle_{q}$, respectively, with $m \in\{1, \ldots, M\}$. Then in the LSL, the joint distribution of $\left(x_{m}, x_{m}^{\prime},\left\langle x_{m}^{\prime}\right\rangle_{q}\right)$ converges to the joint distribution of $\left(x, x^{\prime},\left\langle x^{\prime}\right\rangle_{q}\right)$, associated with scalar Gaussian channels

$$
\begin{aligned}
& z=\sqrt{\rho_{\mathrm{SR}}} x+\frac{w}{\sqrt{\eta_{\mathrm{SR}}}}, \\
& z=\sqrt{\rho_{\mathrm{SR}}} x^{\prime}+\frac{w^{\prime}}{\sqrt{\xi_{\mathrm{SR}}}},
\end{aligned}
$$

where $w, w^{\prime} \sim \mathcal{C N}(0,1)$ and $\left\{\eta_{\mathrm{SR}}, \xi_{\mathrm{SR}}\right\}$ are obtained from a solution of the following system of fixed-point equations

$$
\begin{aligned}
\xi_{\mathrm{RD}} & =\alpha_{\mathrm{RD}}\left(\sigma^{2}+\nu_{\mathrm{RD}}\right)^{-1}, \\
\eta_{\mathrm{RD}} & =\alpha_{\mathrm{RD}}\left(1+\varepsilon_{\mathrm{RD}}\right)^{-1}, \\
\nu_{\mathrm{RD}} & =\frac{\beta \rho_{\mathrm{RD}}\left(\sigma^{2}+\nu_{\mathrm{SR}}\right)}{1+\beta \rho_{\mathrm{RD}} \xi_{\mathrm{RD}}\left(\sigma^{2}+\nu_{\mathrm{SR}}\right)}, \\
\varepsilon_{\mathrm{RD}} & =\frac{\beta \rho_{\mathrm{RD}}\left(1+\varepsilon_{\mathrm{SR}}\right)}{1+\beta \rho_{\mathrm{RD}} \eta_{\mathrm{RD}}\left(1+\varepsilon_{\mathrm{SR}}\right)}, \\
\xi_{\mathrm{SR}} & =\frac{\alpha_{\mathrm{SR}} \beta \rho_{\mathrm{RD}} \xi_{\mathrm{RD}}}{1+\beta \rho_{\mathrm{RD}} \xi_{\mathrm{RD}}\left(\sigma^{2}+\nu_{\mathrm{SR}}\right)}, \\
\eta_{\mathrm{SR}} & =\frac{\alpha_{\mathrm{SR}} \beta \rho_{\mathrm{RD}} \eta_{\mathrm{RD}}}{1+\beta \rho_{\mathrm{RD}} \eta_{\mathrm{RD}}\left(1+\varepsilon_{\mathrm{SR}}\right)}, \\
\nu_{\mathrm{SR}} & =\rho_{\mathrm{SR}} \mathrm{E}_{z, x^{\prime}}\left\{\left|x^{\prime}-\left\langle x^{\prime}\right\rangle\right|^{2}\right\}, \\
\varepsilon_{\mathrm{SR}} & =\rho_{\mathrm{SR}} \mathrm{E}_{z, x}\left\{\left|x-\left\langle x^{\prime}\right\rangle\right|^{2}\right\} .
\end{aligned}
$$

Here, the latter two terms represent the mean square error and posterior variance of channels (7a) and (7b).

Proof: The derivation of the claim is in the Appendix.

The above result reveals that in the LSL, the joint distribution of the input $x_{m}$, postulated input $x_{m}^{\prime}$ and output of 
the GPME $\left\langle x_{m}^{\prime}\right\rangle_{q}$, associated with the MIMO channel (2), converges to the joint distribution of the same set of quantities related to single-input single-output (SISO) Gaussian channels (7a) and (7b). Namely, an AF relay MIMO channel in the LSL decouples into a bank of SISO Gaussian channels. This is known as the decoupling principle previously reported in the context of CDMA systems in [5].

\section{A. Achievable Rates under Separate Decoding}

To realize individually optimal detection, the receiver needs to postulate the actual distribution, i.e., $q\left(x^{\prime}\right)=p(x)$ and $\sigma=$ 1 . This leads to $\xi_{\mathrm{RD}}=\eta_{\mathrm{RD}}, \nu_{\mathrm{RD}}=\varepsilon_{\mathrm{RD}}, \xi_{\mathrm{SR}}=\eta_{\mathrm{SR}}$ and $\nu_{\mathrm{SR}}=\varepsilon_{\mathrm{SR}}$. Moreover, $\varepsilon_{\mathrm{SR}}$ in (8), depending on the input constellation, has to be evaluated numerically (cf. [13]).

Further, the GPME being a strictly increasing decision function [5], the achievable rate under SD becomes

$$
I\left(\left\langle\boldsymbol{x}^{\prime}\right\rangle ; \boldsymbol{x}\right)=-M \ln \frac{\pi \mathrm{e}}{\eta_{\mathrm{SR}}}-M \int p\left(z \mid \eta_{\mathrm{SR}}\right) \ln p\left(z \mid \eta_{\mathrm{SR}}\right) \mathrm{d} z .
$$

It is noteworthy that in this case (8) can have multiple solutions. From those, one should select only the solution minimizing the free energy [8] given as

$$
\begin{aligned}
\mathcal{F}= & I\left(\left\langle\boldsymbol{x}^{\prime}\right\rangle ; \boldsymbol{x}\right)+N \ln \left(1+\varepsilon_{\mathrm{RD}}\right)-M \eta_{\mathrm{SR}} \varepsilon_{\mathrm{SR}}-L \eta_{\mathrm{RD}} \varepsilon_{\mathrm{RD}} \\
& +L \ln \left[1+\beta \rho_{\mathrm{RD}} \eta_{\mathrm{RD}}\left(1+\varepsilon_{\mathrm{SR}}\right)\right]+N(1+\ln \pi) . \quad(10)
\end{aligned}
$$

\section{B. Bit Error Rate}

Since in the LSL the performance of the given system under SD is fully characterized by the equivalent scalar channels (7), the BER of the original system (2) reads as

$$
P_{\mathrm{e}}=Q\left(\sqrt{\rho_{\mathrm{SR}} \eta_{\mathrm{SR}}}\right),
$$

where $\eta_{\mathrm{SR}}$ solves the fixed-point equation system (8). Then, the BER performance of an individually optimal detector is otained by (11), setting $q\left(x^{\prime}\right)=p(x)$ and $\sigma=1$.

To evaluate the performance of a linear detector, one assumes that $q\left(x^{\prime}\right)$ is standard Gaussian. Then, by tuning parameter $\sigma$, performance of the three popular linear equalizers can be evaluated. Namely, the GPME output tends to the output of the MF when $\sigma \rightarrow \infty$, to the output of the ZF filter when $\sigma \rightarrow 0$, and to the output of the linear MMSE filter when $\sigma=1$. Finally, the BER is obtained by (11) after solving (8).

\section{Simulation Results}

In this section, we present the results of numerical simulations. To verify the obtained BER expression (11), we simulate the BER of an AF relay MIMO communication system with QPSK signaling. To satisfy the long term power constraint at the relay, the normalization factor is chosen as $\beta=\left(1+\rho_{\mathrm{SR}}\right)^{-1}$. We fix the numbers of antennas at terminals $M=16, L=28$ and $N=36$ and relation between SNRs at each hop $\rho_{\mathrm{RD}}=0.5 \rho_{\mathrm{SR}}$. Fig. 2 plots the numerical results averaged over 500 channel realizations. We note that the asymptotic result predicts well the behavior of the system at low SNR even for system with finite size. However, as SNR increases it becomes less accurate, and therefore the diversity

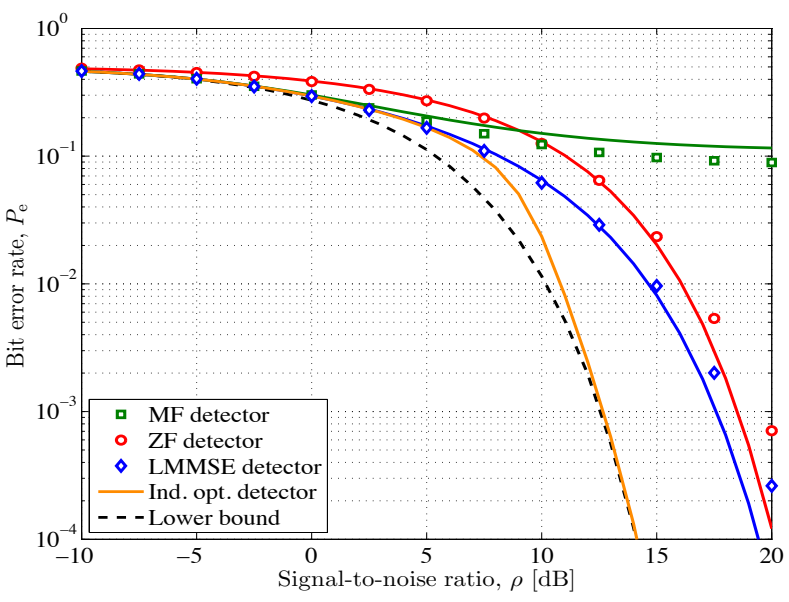

Fig. 2. Average BER vs. SNR for QPSK signaling scheme with detectors. Transmit SNRs for two hops are given by $\rho_{\mathrm{SR}}=\rho$ and $\rho_{\mathrm{RD}}=0.5 \rho$. The numbers of antennas at terminals are set to $M=16, L=28$ and $N=36$. Solid curves denote analytic results, the dashed line denotes the lower bound; markers denote results of Monte Carlo simulations averaged over 500 channel realizations.

order effects, usually visible in that region, are not captured. The reason for this is that at high SNR the approximation becomes much more sensitive to the large-system assumption. It becomes tight as the system size grows infinitely large, while the average BER tends to a Gaussian Q-function.

Meanwhile, Fig. 2 also depicts the average BER of the individually optimal detector. It gradually switches between the performance of the linear MMSE detector and a lower bound, given by

$$
P_{\mathrm{e}}^{\mathrm{LB}}=Q\left(\sqrt{M^{-1} \operatorname{tr}\{\boldsymbol{C}\}}\right),
$$

where $\boldsymbol{C} \triangleq \mathrm{E}_{\mathcal{H}}\left\{\left[\left(\mathbf{I}_{N}+\overline{\boldsymbol{H}}\right)^{-1 / 2} \boldsymbol{H}\right]\left[\left(\mathbf{I}_{N}+\overline{\boldsymbol{H}}\right)^{-1 / 2} \boldsymbol{H}\right]^{\mathrm{H}}\right\}$ is the equivalent signal covariance matrix of the transmission over parallel Gaussian channels.

Fig. 3 plots achievable rates of both SD systems (under individually optimal detection) and JDD (obtained from [8]) are plotted as functions of SNR for different signaling schemes (Gaussian, QPSK and 16-QAM). Note that at certain SNR values, the performance curves of SD with discrete signals suddenly switch to those of JDD. Such a rapid change in performance indicates the occurrence of a phase transition at a certain SNR threshold. A physical analogy to such a behavior is freezing water or the hysteresis of a ferromagnetic material. From the practical point of view, this can be explained by the sparsity of the QPSK constellation, which helps to identify symbols perfectly once the SNR is sufficiently high. Similar behavior was also observed in [6] and [12]. Note also here that for the SD scheme with $\alpha_{\mathrm{SR}}, \alpha_{\mathrm{RD}}>1$, Gaussian signaling is no longer optimal and is outperformed by a discrete constellation, as was previously observed in [14].

\section{CONCLUSIONS}

Using the replica method from statistical physics, we have developed a framework for the asymptotic performance analysis of a dual-hop AF relay MIMO system. The framework captures the effects of separation between detection and decoding, 


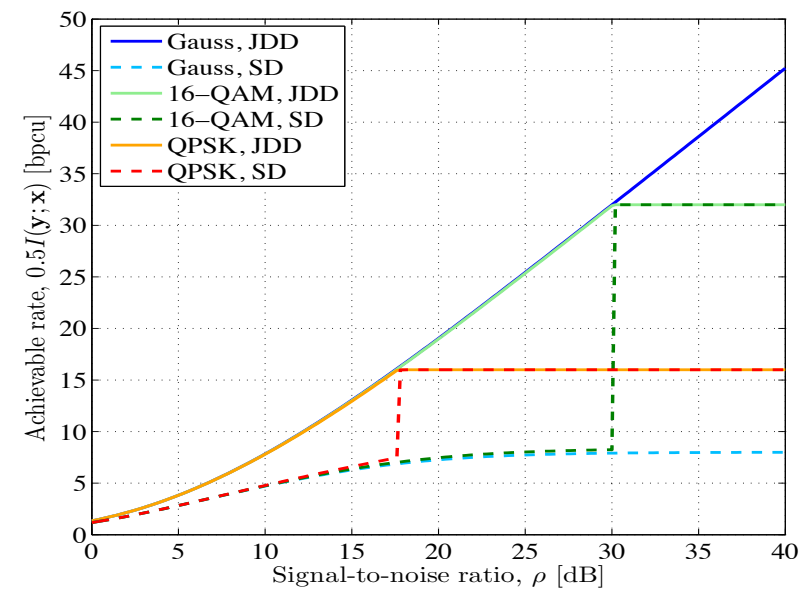

Fig. 3. Achievable ergodic rate $v s$. SNR for various signaling schemes. A factor of 0.5 in front of the rate is due to the half-duplex regime. Transmit SNRs for two hops are given by $\rho_{\mathrm{SR}}=\rho$ and $\rho_{\mathrm{RD}}=0.5 \rho$. Numbers of antennas at terminals are $M=12, L=10, N=8$. Solid curves denote performance of JDD, while dashed lines denote that of SD.

as well as suboptimality of linear detectors. More precisely, we have evaluated the large-system performance of the system under separate detection in terms of achievable ergodic data rate, as well as bit error rate. Comparing to Monte Carlo simulations, it has been confirmed that the results provide an accurate approximation for a finite-sized system at low and moderate SNR. Moreover, the individually optimal detection scheme is shown to experience a phase transition at certain SNR values. The obtained compact expressions are useful for design of coding schemes improving the system performance.

\section{APPENDIX}

\section{DERIVATION OF CLAIM 1}

Consider the channel input, postulated input and output of the GPME for $m, m=1, \ldots, M$. We want to evaluate the joint moments of the joint distribution of $\left(x_{m}, x^{\prime},\left\langle x_{m}^{\prime}\right\rangle_{q}\right)$. For non-negative integers $i_{\mathrm{r}}, i_{\mathrm{i}}, j_{\mathrm{r}}, j_{\mathrm{i}}$, with $b_{\mathrm{r}}, b_{\mathrm{i}} \in\{1, \ldots, u\}, b_{\mathrm{r}} \neq b_{\mathrm{i}}$, let $\mathcal{A}_{\mathrm{r}}$ and $\mathcal{A}_{\mathrm{i}}$ be disjoined subsets of $\{1, \ldots, u\} \backslash\left\{b_{\mathrm{r}}, b_{\mathrm{i}}\right\}$ with cardinalities $l_{\mathrm{r}}$ and $l_{\mathrm{i}}$, respectively. Define a function

$$
\begin{aligned}
g(\boldsymbol{X}) \triangleq & \sum_{m=1}^{M}\left(\operatorname{Re}\left\{x_{m}^{(0)}\right\}\right)^{i_{\mathrm{r}}}\left(\operatorname{Im}\left\{x_{m}^{(0)}\right\}\right)^{i_{\mathrm{i}}}\left(\operatorname{Re}\left\{x_{m}^{\left(b_{\mathrm{r}}\right)}\right\}\right)^{j_{\mathrm{r}}} \\
& \times\left(\operatorname{Im}\left\{x_{m}^{\left(b_{\mathrm{i}}\right)}\right\}\right)^{j_{\mathrm{i}}} \prod_{a_{\mathrm{r}} \in \mathcal{A}_{\mathrm{r}}} \operatorname{Re}\left\{x_{m}^{\left(a_{\mathrm{r}}\right)}\right\} \prod_{a_{\mathrm{i}} \in \mathcal{A}_{\mathrm{i}}} \operatorname{Im}\left\{x_{m}^{\left(a_{\mathrm{i}}\right)}\right\},
\end{aligned}
$$

where $\boldsymbol{X} \triangleq\left[\boldsymbol{x}^{(0) \top}, \ldots, \boldsymbol{x}^{(u) \top}\right]^{\top} \in \mathbb{C}^{M(u+1)}$ with $\boldsymbol{x}^{(0)}$ denoting the original transmitted signal vector $\boldsymbol{x}$, and $\boldsymbol{x}^{(a)}, a \in$ $\{1, \ldots, u\}$ denoting the i.i.d. replicated signal vectors $\boldsymbol{x}^{\prime}$. Let furthermore

$$
Z^{(u)}(\boldsymbol{y}, \mathcal{H}, \boldsymbol{x} ; \omega) \triangleq \mathrm{E}_{\boldsymbol{X}, \boldsymbol{N}_{\mathrm{R}}}\left\{\mathrm{e}^{\omega g(\boldsymbol{X})} \prod_{a=1}^{u} \frac{\mathrm{e}^{-\frac{1}{\sigma^{2}}\left\|\boldsymbol{y}-\boldsymbol{H} \boldsymbol{x}^{(a)}-\overline{\boldsymbol{H}} \boldsymbol{n}_{\mathrm{R}}^{(a)}\right\|^{2}}}{\left(\pi \sigma^{2}\right)^{N}}\right\}
$$

be the partition function of a hypothetic large system, where $\boldsymbol{N}_{\mathrm{R}} \triangleq\left[\boldsymbol{n}_{\mathrm{R}}^{(0)}, \ldots, \boldsymbol{n}_{\mathrm{R}}^{(u)}\right]^{\top} \in \mathbb{C}^{L(u+1)}$ with $\boldsymbol{n}_{\mathrm{R}}^{(0)}$ denoting the noise vector $\boldsymbol{n}_{\mathrm{R}}$ and $\boldsymbol{n}_{\mathrm{R}}^{(a)}$ denoting i.i.d. replicas of the pos- tulated noise $\boldsymbol{n}_{\mathrm{R}}^{\prime}$. Define then a free-energy-like quantity [5]

$$
\mathcal{F}=\left.\frac{1}{M} \lim _{u \rightarrow 0^{+}} \frac{\partial}{\partial \omega} \ln \mathrm{E}_{\boldsymbol{y}, \mathcal{H}, \boldsymbol{x}}\left\{Z^{(u)}(\boldsymbol{y}, \mathcal{H}, \boldsymbol{x} ; \omega)\right\}\right|_{\omega=0},
$$

providing exactly the joint moments of interest.

For the original AF MIMO setup, define a set of vectors $\boldsymbol{v}_{\mathrm{SR}}^{(a)} \triangleq \boldsymbol{H}_{\mathrm{SR}} \boldsymbol{x}^{(a)}+\boldsymbol{n}_{\mathrm{R}}^{(a)}, \boldsymbol{v}_{\mathrm{RD}}^{(a)} \triangleq \sqrt{\beta} \boldsymbol{H}_{\mathrm{RD}} \boldsymbol{v}_{\mathrm{SR}}^{(a)}$, $\boldsymbol{V}_{\mathrm{SR}} \triangleq\left[\boldsymbol{v}_{\mathrm{SR}}^{(0) \mathrm{T}}, \ldots, \boldsymbol{v}_{\mathrm{SR}}^{(u) \mathrm{T}}\right]^{\top} \in \mathbb{C}^{L(u+1)}$ and $\boldsymbol{V}_{\mathrm{RD}} \triangleq$ $\left[\boldsymbol{v}_{\mathrm{RD}}^{(0) \top}, \ldots, \boldsymbol{v}_{\mathrm{RD}}^{(u)]^{\top}} \in \mathbb{C}^{N(u+1)}\right.$. We notice that in the LSL vector $V_{\mathrm{RD}}$ converges to a Gaussian vector with the covariance matrix given by $\boldsymbol{K}_{\mathrm{RD}}=\boldsymbol{Q}_{\mathrm{RD}} \otimes \mathbf{I}_{N}$, where $\left[\boldsymbol{Q}_{\mathrm{RD}}\right]_{a, b}=$ $\frac{\beta \rho_{\mathrm{RD}}}{L} \boldsymbol{v}_{\mathrm{SR}}^{(b) \mathrm{H}} \boldsymbol{v}_{\mathrm{SR}}^{(a)}$ and hence we can rewrite the logarithm in (15)

$M^{-1} \ln \left\{Z^{(u)}(\boldsymbol{y}, \mathcal{H}, \boldsymbol{x} ; \omega)\right\}=M^{-1} \ln \int \mathrm{e}^{N G_{\mathrm{RD}}^{(u)}\left(\boldsymbol{Q}_{\mathrm{RD}}\right)} \mathrm{d} \mu_{\mathrm{RD}}^{(u)}\left(\boldsymbol{Q}_{\mathrm{RD}} ; \omega\right)$,

where the vanishing constants are omitted and function $G_{\mathrm{RD}}^{(u)}\left(\boldsymbol{Q}_{\mathrm{RD}}\right)$ is obtained via the Gaussian integral as

$G_{\mathrm{RD}}^{(u)}\left(\boldsymbol{Q}_{\mathrm{RD}}\right)=-u \ln \pi \sigma^{2}-\ln \left(1+\frac{u}{\sigma^{2}}\right)+\ln \operatorname{det}\left(\mathbf{I}_{u+1}+\boldsymbol{Q}_{\mathrm{RD}} \boldsymbol{\Sigma}\right)$,

with $\boldsymbol{\Sigma} \triangleq \frac{1}{\sigma^{2}\left(\sigma^{2}+u\right)}\left[\begin{array}{cc}u \sigma^{2} & -\sigma^{2} \mathbf{1}_{u}^{\top} \\ -\sigma^{2} \mathbf{1}_{u} & \left(\sigma^{2}+u\right) \mathbf{I}_{u}-\mathbf{1}_{u} \mathbf{1}_{u}^{\top}\end{array}\right]$. The probability measure in (16) is given by

$\mu_{\mathrm{RD}}^{(u)}\left(\boldsymbol{Q}_{\mathrm{RD}} ; \omega\right)=\mathrm{E}_{\boldsymbol{V}_{\mathrm{SR}}}\left\{\mathrm{e}^{\omega g(\boldsymbol{X})} \prod_{a, b=0}^{u} \delta\left(\beta \rho_{\mathrm{RD}} \boldsymbol{v}_{\mathrm{SR}}^{(b) \mathrm{H}} \boldsymbol{v}_{\mathrm{SR}}^{(a)}-L\left[\boldsymbol{Q}_{\mathrm{RD}}\right]_{a, b}\right)\right\}$.

The moment-generating function (MGF) induced by (18) reads

$$
M_{\mathrm{RD}}^{(u)}\left(\tilde{\boldsymbol{Q}}_{\mathrm{RD}} ; \omega\right)=\mathrm{E}_{\boldsymbol{V}_{\mathrm{SR}}}\left\{\mathrm{e}^{\omega g(\boldsymbol{X})} \mathrm{e}^{\beta \rho_{\mathrm{RD}} \boldsymbol{V}_{\mathrm{SR}}^{\mathrm{H}}\left(\tilde{\boldsymbol{Q}}_{\mathrm{RD}} \otimes \mathbf{I}_{L}\right) \boldsymbol{V}_{\mathrm{SR}}}\right\} .
$$

By the large-deviations property [15], in the LSL (18) is dominated by the exponent of the rate function below

$$
I_{\mathrm{RD}}^{(u)}\left(\boldsymbol{Q}_{\mathrm{RD}} ; \omega\right)=\sup _{\tilde{\boldsymbol{Q}}_{\mathrm{RD}}}\left\{\operatorname{tr}\left\{\tilde{\boldsymbol{Q}}_{\mathrm{RD}} \boldsymbol{Q}_{\mathrm{RD}}\right\}-L^{-1} \ln M_{\mathrm{RD}}^{(u)}\left(\tilde{\boldsymbol{Q}}_{\mathrm{RD}} ; \omega\right)\right\} .
$$

Further, by the Gärtner-Ellis theorem [16], in the LSL

$$
\begin{aligned}
M^{-1} \ln \mathrm{E} & \left\{Z^{(u)}(\boldsymbol{y}, \mathcal{H}, \boldsymbol{x} ; \omega)\right\} \\
& =\sup _{\boldsymbol{Q}_{\mathrm{RD}}}\left\{\alpha_{\mathrm{SD}} G_{\mathrm{RD}}^{(u)}\left(\boldsymbol{Q}_{\mathrm{RD}}\right)-\alpha_{\mathrm{SR}} I_{\mathrm{RD}}^{(u)}\left(\boldsymbol{Q}_{\mathrm{RD}} ; \omega\right)\right\} .
\end{aligned}
$$

Next, the term $M^{-1} \ln M_{\mathrm{RD}}^{(u)}\left(\tilde{\boldsymbol{Q}}_{\mathrm{RD}} ; \omega\right)$ as before

$$
M^{-1} \ln M_{\mathrm{RD}}^{(u)}\left(\tilde{\boldsymbol{Q}}_{\mathrm{RD}} ; \omega\right)=M^{-1} \ln \int \mathrm{e}^{L G_{\mathrm{SR}}^{(u)}\left(\boldsymbol{Q}_{\mathrm{SR}}\right)} \mathrm{d} \mu_{\mathrm{SR}}^{(u)}\left(\boldsymbol{Q}_{\mathrm{SR}} ; \omega\right),
$$

with the covariance matrix of vector $\boldsymbol{V}_{\mathrm{SR}}$ being given by $\boldsymbol{K}_{\mathrm{SR}}=\left(\boldsymbol{Q}_{\mathrm{SR}}+\boldsymbol{E}_{\mathrm{SR}}\right) \otimes \mathbf{I}_{L}$, where $\boldsymbol{E}_{\mathrm{SR}} \triangleq\left[\begin{array}{cc}1 & \mathbf{0}_{1 \times u} \\ \mathbf{0}_{u \times 1} & \sigma^{2} \mathbf{I}_{u}\end{array}\right]$, and $\left[\boldsymbol{Q}_{\mathrm{SR}}\right]_{a, b}=\frac{\rho}{M} \boldsymbol{x}^{(b) \mathrm{H}} \boldsymbol{x}^{(a)}$. Function $G_{\mathrm{SR}}^{(u)}\left(\boldsymbol{Q}_{\mathrm{SR}}\right)$ is again obtained through the Gaussian integral yielding

$$
G_{\mathrm{SR}}^{(u)}\left(\boldsymbol{Q}_{\mathrm{SR}}\right)=-\ln \operatorname{det}\left[\mathbf{I}_{(u+1)}-\beta \rho_{\mathrm{RD}} \tilde{\boldsymbol{Q}}_{\mathrm{RD}}\left(\boldsymbol{Q}_{\mathrm{SR}}+\boldsymbol{E}_{\mathrm{SR}}\right)\right] .
$$

Meanwhile, the probability measure for $Q_{\mathrm{SR}}$ reads

$$
\mu_{\mathrm{SR}}^{(u)}\left(\boldsymbol{Q}_{\mathrm{SR}} ; \omega\right)=\mathrm{E}_{\boldsymbol{X}}\left\{\mathrm{e}^{\omega g(\boldsymbol{X})} \prod_{a, b=0}^{u} \delta\left(\beta \rho_{\mathrm{RD}} \boldsymbol{x}^{(b){ }^{\mathrm{H}}} \boldsymbol{x}^{(a)}-M\left[\boldsymbol{Q}_{\mathrm{SR}}\right]_{a, b}\right)\right\} .
$$




$$
\begin{aligned}
& T_{\mathrm{SR}}^{(u)}(\mathcal{Q}, \tilde{\mathcal{Q}} ; \omega)=\tilde{r}_{\mathrm{SR}} r_{\mathrm{SR}}+u \tilde{m}_{\mathrm{SR}} m_{\mathrm{SR}}+u \tilde{m}_{\mathrm{SR}}^{*} m_{\mathrm{SR}}^{*}+u \tilde{p}_{\mathrm{SR}} p_{\mathrm{SR}}+u(u-1) \tilde{q}_{\mathrm{SR}} q_{\mathrm{SR}}+\alpha_{\mathrm{SD}} u \ln \pi \\
& +\alpha_{\mathrm{SR}}\left(\tilde{r}_{\mathrm{RD}} r_{\mathrm{RD}}+u \tilde{m}_{\mathrm{RD}} m_{\mathrm{RD}}+u \tilde{m}_{\mathrm{RD}}^{*} m_{\mathrm{RD}}^{*}+u \tilde{p}_{\mathrm{RD}} p_{\mathrm{RD}}+u(u-1) \tilde{q}_{\mathrm{RD}} q_{\mathrm{RD}}\right)+(u-1) \alpha_{\mathrm{SD}} \ln \left(\sigma^{2}+p_{\mathrm{RD}}-q_{\mathrm{RD}}\right) \\
& +\alpha_{\mathrm{SD}} \ln \left[\sigma^{2}+p_{\mathrm{RD}}-q_{\mathrm{RD}}+u\left(1+r_{\mathrm{RD}}-m_{\mathrm{RD}}-m_{\mathrm{RD}}^{*}+q_{\mathrm{RD}}\right)\right]+(u-1) \alpha_{\mathrm{SR}} \ln \left[1-\beta \rho_{\mathrm{RD}}\left(\tilde{p}_{\mathrm{RD}}-\tilde{q}_{\mathrm{RD}}\right)\left(\sigma^{2}+p_{\mathrm{SR}}-q_{\mathrm{SR}}\right)\right] \\
& +\alpha_{\mathrm{SR}} \ln \left(1-\beta \rho_{\mathrm{RD}}\left[u \tilde{m}_{\mathrm{RD}}^{*} m_{\mathrm{SR}}+u \tilde{m}_{\mathrm{RD}} m_{\mathrm{SR}}^{*}+\left(\tilde{p}_{\mathrm{RD}}+(u-1) \tilde{q}_{\mathrm{RD}}\right)\left(\sigma^{2}+p_{\mathrm{SR}}+(u-1) q_{\mathrm{SR}}\right)+\tilde{r}_{\mathrm{RD}}\left(1+r_{\mathrm{SR}}\right)\right]\right. \\
& \left.+\beta^{2} \rho_{\mathrm{RD}}^{2}\left[u\left|\tilde{m}_{\mathrm{RD}}\right|^{2}-\left(1+r_{\mathrm{SR}}\right)\left(\sigma^{2}+p_{\mathrm{SR}}+(u-1) q_{\mathrm{SR}}\right)\right]\left[u\left|m_{\mathrm{SR}}\right|^{2}-\tilde{r}_{\mathrm{RD}}\left(\tilde{p}_{\mathrm{RD}}+(u-1) \tilde{q}_{\mathrm{RD}}\right)\right]\right) \\
& -\ln \frac{\eta_{\mathrm{SR}}}{\pi}-\frac{1}{M} \ln \int \mathrm{E}_{\boldsymbol{X}}\left\{\mathrm{e}^{\omega g(\boldsymbol{X})} \mathrm{e}^{-\eta_{\mathrm{SR}}\left\|\boldsymbol{z}-\sqrt{\rho_{\mathrm{SR}}} \boldsymbol{x}\right\|^{2}} \mathrm{e}^{\rho_{\mathrm{SR}} \phi_{\mathrm{SR}} \boldsymbol{x}^{\mathrm{H}} \boldsymbol{x}}\left[\mathrm{e}^{-\xi_{\mathrm{SR}}\left\|\boldsymbol{z}-\sqrt{\rho_{\mathrm{SR}}} \boldsymbol{x}^{\prime}\right\|^{2}} \mathrm{e}^{\xi_{\mathrm{SR}} \boldsymbol{z}^{\mathrm{H}} \boldsymbol{z}+\rho_{\mathrm{SR}} \psi_{\mathrm{SR}} \boldsymbol{x}^{\prime \mathrm{H}} \boldsymbol{x}^{\prime}}\right]^{u}\right\} \mathrm{d} \boldsymbol{z}
\end{aligned}
$$

The corresponding MGF is given by

$$
M_{\mathrm{SR}}^{(u)}\left(\tilde{\boldsymbol{Q}}_{\mathrm{SR}} ; \omega\right)=\mathrm{E}_{\boldsymbol{X}}\left\{\mathrm{e}^{\rho_{\mathrm{SR}} \boldsymbol{X}^{\mathrm{H}}\left(\tilde{\boldsymbol{Q}}_{\mathrm{SR}} \otimes \mathbf{I}_{M}\right) \boldsymbol{X}}\right\} .
$$

Again, measure $\mu_{\mathrm{SR}}^{(u)}\left(\boldsymbol{Q}_{\mathrm{RD}}\right)$ is dominated by the exponent of

$$
I_{\mathrm{SR}}^{(u)}\left(\boldsymbol{Q}_{\mathrm{SR}} ; \omega\right)=\sup _{\tilde{\boldsymbol{Q}}_{\mathrm{SR}}}\left\{\operatorname{tr}\left\{\tilde{\boldsymbol{Q}}_{\mathrm{SR}} \boldsymbol{Q}_{\mathrm{SR}}\right\}-M^{-1} \ln M_{\mathrm{SR}}^{(u)}\left(\tilde{\boldsymbol{Q}}_{\mathrm{SR}} ; \omega\right)\right\},
$$

and by the Gärtner-Ellis theorem we have

$$
M^{-1} \ln \mathrm{E}\left\{Z^{(u)}(\boldsymbol{y}, \mathcal{H}, \boldsymbol{x} ; \omega)\right\}=\inf _{\mathcal{Q}} \sup _{\tilde{\mathcal{Q}}}\left\{T_{\mathrm{SR}}^{(u)}(\mathcal{Q}, \tilde{\mathcal{Q}} ; \omega)\right\},
$$

where $\mathcal{Q} \triangleq\left\{\boldsymbol{Q}_{\mathrm{SR}}, \boldsymbol{Q}_{\mathrm{RD}}\right\}, \tilde{\mathcal{Q}} \triangleq\left\{\tilde{\boldsymbol{Q}}_{\mathrm{SR}}, \tilde{\boldsymbol{Q}}_{\mathrm{RD}}\right\}$ and

$$
\begin{aligned}
& T_{\mathrm{SR}}^{(u)}(\mathcal{Q}, \tilde{\mathcal{Q}} ; \omega)=-M^{-1} \ln \mathrm{E}_{\boldsymbol{X}}\left\{\mathrm{e}^{\omega g(\boldsymbol{X})} \mathrm{e}^{\rho_{\mathrm{SR}} \boldsymbol{X}^{\mathrm{H}}\left(\tilde{\boldsymbol{Q}}_{\mathrm{SR}} \otimes \mathbf{I}_{M}\right) \boldsymbol{X}}\right\} \\
& \quad+\alpha_{\mathrm{SD}} \ln \left(1+\frac{u}{\sigma^{2}}\right)+\alpha_{\mathrm{SD}} \ln \operatorname{det}\left(\mathbf{I}_{u+1}+\boldsymbol{Q}_{\mathrm{RD}} \boldsymbol{\Sigma}\right) \\
& \quad+\alpha_{\mathrm{SR}} \ln \operatorname{det}\left[\mathbf{I}_{L}-\beta \rho_{\mathrm{RD}} \tilde{\boldsymbol{Q}}_{\mathrm{RD}}\left(\boldsymbol{Q}_{\mathrm{SR}}+\boldsymbol{E}_{\mathrm{SR}}\right)\right] \\
& \quad+\operatorname{tr}\left\{\tilde{\boldsymbol{Q}}_{\mathrm{SR}} \boldsymbol{Q}_{\mathrm{SR}}\right\}+\alpha_{\mathrm{SR}} \operatorname{tr}\left\{\tilde{\boldsymbol{Q}}_{\mathrm{RD}} \boldsymbol{Q}_{\mathrm{RD}}\right\}+\alpha_{\mathrm{SD}} u \ln \pi \sigma^{2} .
\end{aligned}
$$

The corresponding optimization problem is prohibitively hard to solve in general and hence we invoke the simplifying replica symmetry (RS) assumption, by postulating the following structure for matrices $Q_{\mathrm{SR}}, \tilde{Q}_{\mathrm{SR}}, Q_{\mathrm{RD}}$ and $\tilde{Q}_{\mathrm{RD}}$ :

$$
\boldsymbol{Q}=\left[\begin{array}{cc}
r & m \mathbf{1}_{u}^{\top} \\
m^{*} \mathbf{1}_{u} & (p-q) \mathbf{I}_{u}+q \mathbf{1}_{u} \mathbf{1}_{u}^{\top}
\end{array}\right], \tilde{\boldsymbol{Q}}=\left[\begin{array}{cc}
\tilde{r} & \tilde{m} \mathbf{1}_{u}^{\top} \\
\tilde{m}^{*} \mathbf{1}_{u} & (\tilde{p}-\tilde{q}) \mathbf{I}_{u}+\tilde{q} \mathbf{1}_{u} \mathbf{1}_{u}^{\top}
\end{array}\right] .
$$

With the RS assumption above (keeping in mind that $\boldsymbol{x}^{(0)}=$ $\boldsymbol{x}$ and $\boldsymbol{x}^{(a)}=\boldsymbol{x}^{\prime}, \forall a$ ) we can simplify (28) as in (31) on the top of the page, where we defined $\eta_{\mathrm{SR}} \triangleq \frac{\left|\tilde{m}_{\mathrm{SR}}\right|^{2}}{\tilde{q}_{\mathrm{SR}}}, \phi_{\mathrm{SR}} \triangleq \tilde{r}_{\mathrm{SR}}$, $\xi_{\mathrm{SR}} \triangleq \tilde{m}_{\mathrm{SR}}^{*}$ and $\psi_{\mathrm{SR}} \triangleq \tilde{m}_{\mathrm{SR}}^{*}+\tilde{p}_{\mathrm{SR}}-\tilde{q}_{\mathrm{SR}}$. The last term of (31) is thus associated with the two Gaussian scalar channels in (7).

Now, to find the saddle point, we take the derivatives of (31) w.r.t. to all the 16 parameters, which yields $\tilde{r}_{\mathrm{SR}}=0, \tilde{r}_{\mathrm{RD}}=0$, $\tilde{m}_{\mathrm{SR}}^{*}=\tilde{m}_{\mathrm{SR}}, \tilde{m}_{\mathrm{RD}}^{*}=\tilde{m}_{\mathrm{RD}}, \tilde{p}_{\mathrm{SR}}-\tilde{q}_{\mathrm{SR}}=-\tilde{m}_{\mathrm{SR}}$, as well as

$$
\begin{aligned}
r_{\mathrm{SR}}-m_{\mathrm{SR}}-m_{\mathrm{SR}}^{*}+q_{\mathrm{SR}} & =\rho \mathrm{E}_{z, x}\left\{\left|x-\left\langle x^{\prime}\right\rangle\right|^{2}\right\}, \\
p_{\mathrm{SR}}-q_{\mathrm{SR}} & =\rho \mathrm{E}_{z, x^{\prime}}\left\{\left|x^{\prime}-\left\langle x^{\prime}\right\rangle\right|^{2}\right\},
\end{aligned}
$$

thus obtaining the set of fixed-point equations (8), where $\varepsilon_{\mathrm{SR}} \triangleq r_{\mathrm{SR}}-m_{\mathrm{SR}}-m_{\mathrm{SR}}^{*}+q_{\mathrm{SR}}, \varepsilon_{\mathrm{RD}} \triangleq r_{\mathrm{RD}}-m_{\mathrm{RD}}-$ $m_{\mathrm{RD}}^{*}+q_{\mathrm{RD}}, \nu_{\mathrm{SR}} \triangleq p_{\mathrm{SR}}-q_{\mathrm{SR}}$ and $\nu_{\mathrm{RD}} \triangleq p_{\mathrm{RD}}-q_{\mathrm{RD}}$.

We notice that $\omega$ is present only in the last part of (31). Therefore, the free energy is obtained by (15) as

$$
\mathcal{F}=\int p_{i_{\mathrm{r}}, i_{\mathrm{i}}}\left(z ; \eta_{\mathrm{SR}}\right) \frac{q_{j_{\mathrm{r}}, j_{\mathrm{i}}}\left(z ; \xi_{\mathrm{SR}}\right)}{q_{0,0}\left(z ; \xi_{\mathrm{SR}}\right)}\left[\frac{q_{1,0}\left(z ; \xi_{\mathrm{SR}}\right)}{q_{0,0}\left(z ; \xi_{\mathrm{SR}}\right)}\right]^{l_{\mathrm{r}}}\left[\frac{q_{0,1}\left(z ; \xi_{\mathrm{SR}}\right)}{q_{0,0}\left(z ; \xi_{\mathrm{SR}}\right)}\right]_{(33)}^{l_{\mathrm{i}}} \mathrm{d} z
$$

where $p_{i_{\mathrm{r}}, i_{\mathrm{i}}}\left(z ; \eta_{\mathrm{SR}}\right) \triangleq \mathrm{E}_{\boldsymbol{x}}\left\{(\operatorname{Re}\{x\})^{i_{\mathrm{r}}}(\operatorname{Im}\{x\})^{i_{\mathrm{i}}} p\left(z \mid x ; \eta_{\mathrm{SR}}\right)\right\}$ and $q_{j_{\mathrm{r}}, j_{\mathrm{i}}}\left(z ; \xi_{\mathrm{SR}}\right) \triangleq \mathrm{E}_{\boldsymbol{x}^{\prime}}\left\{\left(\operatorname{Re}\left\{x^{\prime}\right\}\right)^{j_{\mathrm{r}}}\left(\operatorname{Im}\left\{x^{\prime}\right\}\right)^{j_{\mathrm{i}}} q\left(z \mid x^{\prime} ; \xi_{\mathrm{SR}}\right)\right\}$.

The above expression reduces to the joint moments of the joint distribution of $\left(x, x^{\prime},\left\langle x^{\prime}\right\rangle_{q}\right)$, which, by the Carleman theorem, implies convergence in distributions [5].

\section{REFERENCES}

[1] E. Telatar, "Capacity of multi-antenna Gaussian channels," European Trans. Telecommun., vol. 10, no. 6, pp. 585-595, 1999.

[2] J. N. Laneman, D. N. Tse, and G. W. Wornell, "Cooperative diversity in wireless networks: Efficient protocols and outage behavior," IEEE Trans. Inf. Theory, vol. 50, no. 12, pp. 3062-3080, 2004.

[3] D. Gesbert, S. Hanly, H. Huang, S. Shamai Shitz, O. Simeone, and W. Yu, "Multi-cell MIMO cooperative networks: A new look at interference," IEEE J. Sel. Areas Commun., vol. 28, no. 9, pp. 1380-1408, 2010.

[4] T. Cover and A. El Gamal, "Capacity theorems for the relay channel," IEEE Trans. Inf. Theory, vol. 25, no. 5, pp. 572-584, 1979.

[5] D. Guo and S. Verdú, "Randomly spread CDMA: Asymptotics via statistical physics," IEEE Trans. Inf. Theory, vol. 51, no. 6, pp. 19832010, Jun. 2005.

[6] R. R. Müller, "Channel capacity and minimum probability of error in large dual antenna array systems with binary modulation," IEEE Trans. Signal Process., vol. 51, no. 11, pp. 2821-2828, Nov. 2003.

[7] C.-K. Wen and K.-K. Wong, "On the sum-rate of uplink MIMO cellular systems with amplify-and-forward relaying and collaborative base stations," IEEE J. Sel. Areas Commun., vol. 28, no. 9, pp. 14091424,2010

[8] M. A. Girnyk, M. Vehkaperä, and L. K. Rasmussen, "Large-system analysis of the $K$-hop AF MIMO relay channel with arbitrary inputs," in Proc. IEEE Int. Symp. Inf. Theory (ISIT), 2013, pp. 439-443.

[9] M. A. Girnyk and L. K. Rasmussen, "Myopic multi-hop transmission strategies in layered wireless networks," in Proc. IEEE Int. Symp. Pers. Indoor Mobile Radio Commun. (PIMRC), 2011, pp. 1763-1767.

[10] I. Trigui, S. Affes, and A. Stephenne, "Closed-form error analysis of variable-gain multihop systems in Nakagami- $m$ fading channels," IEEE Trans. Commun., vol. 59, no. 8, pp. 2285-2295, 2011.

[11] H. Kim and C. Tepedelenlioglu, "Performance bounds on average error rates using the AM-GM inequality and their applications in relay networks," IEEE Trans. Wireless Commun., vol. 11, no. 8, pp. 29862995, 2012.

[12] T. Tanaka, "A statistical-mechanics approach to large-system analysis of CDMA multiuser detectors," IEEE Trans. Inf. Theory, vol. 48, no. 11, pp. 2888-2910, Nov. 2002.

[13] A. Lozano, A. M. Tulino, and S. Verdú, "Optimum power allocation for parallel Gaussian channels with arbitrary input distributions," IEEE Trans. Inf. Theory, vol. 52, no. 7, pp. 3033-3051, 2006.

[14] R. R. Müller and W. H. Gerstacker, "On the capacity loss due to separation of detection and decoding," IEEE Trans. Inf. Theory, vol. 50, no. 8, pp. 1769-1778, 2004.

[15] R. Ellis, Entropy, large deviations, and statistical mechanics. Taylor \& Francis US, 2005, vol. 1431, no. 821.

[16] P. Billingsley, Probability and Measure, 3rd ed. Hoboken, NJ: John Wiley and Sons, Inc., 1995. 